\title{
28 Resarach Soure \\ Endoplasmic Reticulum Stress-related \\ Classification for Prognosis Prediction in Hepatocellular Carcinoma
}

\section{Genhao Zhang ( $\sim$ wangshuya617@bjmu.edu.cn )}

Zhengzhou University First Affiliated Hospital https://orcid.org/0000-0002-9834-6477

\section{Research Article}

Keywords: HCC, prognosis, immune, ER stress, signature

Posted Date: August 24th, 2021

DOl: https://doi.org/10.21203/rs.3.rs-818967/v1

License: (c) (1) This work is licensed under a Creative Commons Attribution 4.0 International License.

Read Full License 


\section{Abstract}

Background: Cancer cells under ER stress are common in hepatocellular carcinoma (HCC) and ER stress is strongly associated with poor prognosis. The aim of this study was to discover credible biomarkers for predicting prognosis of HCC based on ER stress-related genes (ERSRGs).

Methods: Univariate Cox regression was performed to calculate the association between ERSRGs and survival outcomes of HCC patients in TCGA. Then LASSO-Cox regression strategy and stepwise Cox regression examination were performed to investigate the quality and establish the prognostic characteristics associated with prognosis. Finally, the model was subsequently validated in two additional independent HCC cohorts.

Results: A novel seven-gene prognostic risk model based on ERSRGs was constructed and exhibited superior accuracy in forecasting the survival outcomes and 1-, 2-, 3-year survival rate of HCC patients. qRT-PCR was performed to validate the prognostic risk model in an independent clinical cohort containing $59 \mathrm{HCC}$ patients and the results revealed that this signature had a good prognostic performance. Moreover, we found ER stress could affect the immune microenvironment in HCC and immune checkpoint inhibitors (ICls) treatment was more effective for patients in high-risk subgroup. In addition, we identified 103 tumor-sensitive drugs in the CellMiner database that may be available for the treatment of HCC patients targeting ER stress and constructed a nomogram combining ER stress-related feature, TNM stage, age and gender.

Conclusions: Our seven genetic risk model associated with ER stress can accurately predict survival outcome in HCC patients and facilitate the selection of the best individualized treatment targeting ER stress.

\section{Introduction}

The endoplasmic reticulum (ER) is a multifunctional organelle consisting of branching tubes and flattened vesicles that is the main site of protein synthesis and transport, lipid biosynthesis and calcium storage[1, 2]. However, many factors, including inhibition of protein glycosylation, oxidative stress, nutritional deficiencies, imbalance of calcium homeostasis and hypoxia, could reduce the efficiency of ER in processing protein folding and finally lead to ER stress and unresponsive protein response (UPR) $[3,4]$. UPR plays a crucial role in regulating cellular adaptation to ER stress by increasing ER content, improving ER protein folding capacity and downgrading misfolded proteins $[5,6]$. Three transmembrane ER sensors, including activating transcription factor 6 (ATF6), protein kinase $R$ (PKR)-like endoplasmic reticulum kinase (PERK) and inositol-requiring enzyme 1 (IRE1a), have been found to determine the triggering of ER stress and subsequent activation of the UPR[7]. With the increasing recognition of ER stress mechanisms, ER stress dysregulation has been found to play an essential role in various human diseases, including cardiometabolic diseases[8-10], diabetes[11, 12], chronic kidney disease[13, 14], Alzheimer's disease[15, 16] and cancers[17-20]. High levels of ER in hepatocytes can rhythmically activate the hepatic UPR to 
regulate lipid and glucose metabolism, while ER stress and chronic activation of the UPR due to hepatic viral infection or obesity could cause liver dysfunction and metabolic disorders[21]. ER stress plays a crucial role in the pathogenesis of nonalcoholic fatty liver disease (NAFLD)[22] and is strongly associated with survival and death in HCC patients[23]. Recent studies suggested that ER stress and UPR have emerged as new signaling targets for therapeutic interventions in NAFLD and $\mathrm{HCC}[3,24-26]$. In the current study, a novel prognostic risk model based on ER stress-related genes (ERSRGs) was constructed, which could be effectively used for prognostic classification of HCC patients and utilized as a potential target for individualized immunotherapy.

\section{Materials And Methods}

\subsection{Public datasets and generation of ERSRGs}

This study included mRNA expression data and clinical features of HCC patients from two publicly available datasets including TCGA-LIHC and ICGC (LIRI-JP). ERSRGs were available from previous research[4].

\subsection{Prognostic risk score model construction and Functional Analysis}

The univariable cox relapses were to begin with performed to calculate the affiliation between ERSRGs and survival results of HCC patients in two cohorts. LASSO-Cox relapse strategy and stepwise Cox relapse examination were at that point performed to survey the over covering prognosis-related qualities and set up prognostic characteristics. Risk score was at last set up based on the premise of directly combining the equation underneath with the mRNA expression level duplicated the multivariate Cox relapse coefficient $(\beta)$ demonstrate. Risk score $=(\beta m R N A 1 \times$ mRNA1 $)+(\beta m R N A 2 \times$ mRNA2 $)+\ldots+$ $(\beta m R N A n \times m R N A n)$. We stratified patients in TCGA dataset into two subgroups due to ideal hazard score edge. The prescient control and autonomy of the prognostic signature in TCGA were evaluated by ROC examination, Kaplan-Meier survival examination and cox relative risks relapse investigation. Gene set enrichment analysis (GSEA) between the two subgroups was performed to distinguish the altogether cautioned GO items with FDR $<0.05$.

\subsection{Clinical specimens and Quantitative real-time PCR (qRT-PCR) analysis}

Fresh frozen tumor tissues from previously collected HCC patients were selected as an independent validation cohort [27]. qRT-PCR was used to detect the mRNA levels of genes in the model [28]. After the relative mRNAs expression levels were normalized to $\beta$-ACTIN and $\log _{2}$ transformed, patients were stratified into two subgroups according to the above formula. Primer sequences are showed in Table S1.

\subsection{Immune status calculation and immune infiltrates analysis}

The immune status of each sample was assessed by applying the ESTIMATE algorithm to the TCGA cohort and calculating immune and stromal scores. Association between risk scores and immune, 
stromal scores were analyzed by Pearson correlation analysis. To explore impacts of the prognostic model on immunotherapies, we calculated the relationship among risk score and 15 potentially available targeted immune checkpoint genes[29]. Furthermore, in order to assess the potential association between prognostic signature and tumor-infiltrating immune cells (TIICs) in HCC microenvironment, TCGA database was used to measure the abundance ratios of 22 types of TIICs through CIBERSORT[30] (http://cibersort.stanford.edu/). Finally, the predictive ability of significantly changed TIICs was assessed by Kaplan-Meier survival analysis.

\subsection{Genetic alterations and TMB analysis}

The mutation and CNA data of 350 HCC patients were downloaded from TCGA to analyze the difference of genetic alterations between the high- and low- risk score subgroups with R package "maftools", and the tumor mutation burden (TMB) of each patient was subsequently assessed.

\subsection{Drug susceptibility analysis}

The association between anticancer drug sensitivity and mRNA molecules in our risk model was directly explored in the CellMiner database[31]. 574 in advanced clinical trials and 216 Food and Drug Administration (FDA)-approved drugs were used for follow-up analyses. Drugs with adjusted $P$ value $<0.001$ and Pearson correlation coefficient $>0.3$ as cut-off criteria were considered tumor-sensitive drugs.

\subsection{Statistical analysis}

Categorical data were compared with Pearson chi-square test or Fisher exact test whenever appropriate, and quantitative variables were analyzed using independent-samples $t$ test. ROC curve analysis and Kaplan-Meier survival analysis were performed to assess the prediction performance of survival outcomes with $\mathrm{R}$ software (Version 4.0.3). Cox proportional model was performed to analyze relationship between prognostic signature and survival outcomes, together with other clinical features. Clinical characteristics of HCC patients in TCGA, ICGC and clinical validation cohorts were showed in Table S2. Results were considered statistically significant when $P$ value $<0.05$.

\section{Results}

\subsection{Identification of overlapped ERSRGs in TCGA and ICGC cohorts}

As calculated by univariable cox regressions with an adjust $P$ value $<0.05,330$ ERSRGs in TCGA and 225 ERSRGs in ICGC had significant prognostic relevance, respectively. Then 111 genes were identified as overlapped ERSRGs for further analysis (Figure 1A). The expression levels of the 111 overlapping ERSRGs in normal and tumor tissues in TCGA-LIHC dataset were shown in Figure S1A. Results of Go and KEGG analysis indicated that these overlapped genes were majorly associated with ER stress-related pathway (Figure S1B).

\subsection{Establishment of an ER stress-related signature in TCGA}


Overlapped ERSRGs were selected by performing the LASSO-Cox regression model based on the minimum value of $\lambda$ and 19 genes were screened as shown in Figure 1B. These 19 genes were then placed into a stepwise Cox proportional model and finally a prognostic seven-gene signature was identified. Risk score $=(0.17243378 \times$ MAPT $)+(0.09459892 \times N Q 01)-(0.12755413 \times P O N 1)-(0$. $14029120 \times$ PPARGC1A $)+(0.65200737 \times N G L Y 1)+(0.37755837 \times C D K 5)+(0.19417209 \times R N F 186)$. Risk scores for HCC patients were calculated with the above formula, and patients were stratified into high- or low-risk subgroups with an optimal risk score threshold (Figure $2 \mathrm{C}$ ). The association between risk score and clinical characteristics including age, gender, grade, stage, vascular invasion, value of AFP, cirrhosis, HBV infection status and tumor status were evaluated. The results revealed that higher risk scores were linked to advanced TNM stage, later grade, later T stage, HBV infection and recurrence (Supplementary Figure S2). Kaplan-Meier survival analysis revealed that patients with higher risk score were significantly relevant to poorer survival outcomes (Figure 2D). In addition, further stratified survival analysis was applied for different clinical characteristics, and the results demonstrated that this prognostic model could further differentiated patients with different clinical characteristics including age, vascular invasion, grade, recurrence, TNM stage, gender, HBV infection status and AFP value (Supplementary Figure S3). Finally, ROC analysis revealed that this signature had a good prognostic performance with AUCs at 1-, 2-, 3 -year of $0.800,0.732,0.745$ (Figure 2E). To explore whether the seven-gene signature could be acted as an independent prognostic model for HCC patients, univariable and multivariate Cox analysis were performed. Univariable Cox regression analysis revealed that this seven -gene signature was statistically associated with survival outcomes for $\mathrm{HCC}$ patients $(\mathrm{HR}=3.088,95 \% \mathrm{Cl} 2.200-4.335, P<0.001$, Figure $2 \mathrm{~F}$ Left). Then statistically significant variables obtained above were entered into multivariate Cox regression analysis, which revealed that this signature could be served as an independent prognostic factor for HCC patients (HR=2.203, 95\% Cl 1.313-3.694, $P<0.001$, Figure 2F Right) after adjusting for other clinical features.

\subsection{Verification of the signature in ICGC cohort}

To validate the signature, ICGC dataset was applied as a validation cohort. Risk scores of patients were calculated with the same formula, and patients were stratified into high- or low-risk subgroups in ICGC cohort (Figure 3A). Kaplan-Meier survival analysis revealed that patients with higher risk score were prominently relevant to poorer OS rate in ICGC cohort (Figure 3B). ROC analysis revealed that this signature had a good prognostic performance with AUCs at 1-, 2-, 3-year of 0.723, 0.740, 0.736 in ICGC cohort (Figure 3C). To explore whether the seven-gene signature could be acted as an independent prognostic model for HCC patients, univariable and multivariate Cox analysis were performed. Univariable Cox regression analysis revealed that this seven -gene signature was statistically associated with survival outcomes for $\mathrm{HCC}$ patients ( $\mathrm{HR}=3.054,95 \% \mathrm{Cl} 1.882-4.958, P<0.001$, Figure 3D Left). Then statistically significant variables obtained above were entered into multivariate Cox regression analysis, which revealed that this signature could be served as an independent prognostic factor for HCC patients $(\mathrm{HR}=2.463,95 \% \mathrm{Cl} 1.487-4.078, P<0.001$, Figure 3D Right) after adjusting for other clinical features.

\subsection{Functional analysis and Immune infiltrates analysis}


Five Go items with FDR < 0.05 were enriched in this signature, including adaptive immune response, defense response to virus, immune effector process, response to virus and T cell differentiation (Figure 3A). According to the results of ESTIMATE algorithm, risk scores was significantly associated with stromal scores, but not immune scores (Figure 3B), and patients in low-risk subgroup had higher stromal scores when compared with patients in high-risk subgroup (Figure 3C), indicating that this signature was closely related to tumor immune status. Furthermore, based on CIBERSORT algorithm, the differences of 22 types of TIICs in two subgroups in TCGA were assessed by Wilcoxon signed-rank test analysis, and the results demonstrated that HCC patients in low-risk score subgroup had modestly increased ratios of resting mast cells, while patients in high-risk score subgroup had significantly elevated ratios of Macrophages.M0 cells (Figure 3D). Furthermore, Kaplan-Meier survival analysis revealed that only Macrophages.MO cells were prominently relevant to poor survival outcomes in HCC patients (Figure 3E).

\subsection{Genetic alterations and TMB analysis}

The results of genetic alterations analysis indicated that the mutation rates of the top 10 most significantly mutated genes were remarkably different in the two subgroups (Figure 4A\&4B). Subsequently, the TMB of each patient was assessed. We found that the risk score was closely related with TMB and patients in high-risk scores subgroup had significantly increased TMB (Figure 4C).

\subsection{Immune checkpoint genes analysis}

In the following, the expression levels of 15 potentially targetable immune checkpoint genes were compared between the two subgroups in TCGA database, and results showed that patients in high-risk subgroup had significantly increased PD1, PD-L1, CCL2, LAG3, CD276, CTLA4, CXCR4, IL1A, PD-L2, TGFB1, OX40 and CD137 (Figure 5), indicating that immune checkpoint inhibitors (ICls) treatment was more effective for patients in high-risk subgroup.

\subsection{Establishment of a nomogram model in TCGA}

To investigate the coefficient prediction efficiency of this signature, a nomogram model was established in TCGA dataset, and the result revealed that the nomogram with a C-index of 0.723 could help us provide a quantitative method for predicting the 1-, 2-, 3-year survival rate accurately (Figure 6A). The overlap between the forecasted and actual probabilities of 1-, 2-, 3-year survival rate in the calibration curves indicated good agreement (Figure 6B).

\subsection{Drug susceptibility analysis}

Among the 574 in advanced clinical trials and 216 Food and Drug Administration (FDA)-approved drugs, 103 were considered tumor-sensitive drugs (Table S3) and the top 16 most significant tumor-sensitive drugs were shown in Figure 7.

\subsection{Expression levels of genes in risk model}


The mRNA expression of the seven genes in HCC samples were explored in the independent clinical cohort by qRT-PCR (Figure 8A). Risk scores of patients were calculated with the same formula, and patients were stratified into high- or low-risk subgroups (Figure 8A). Kaplan-Meier survival analysis revealed that patients with higher risk score were prominently relevant to poorer OS rate (Figure 8B). ROC analysis revealed that this signature had a good prognostic performance with AUCs at 1-, 2-, 3-year of $0.846,0.824,0.686$ (Figure 8C).

\section{Discussion}

There is growing evidence that ER stress-mediated cell proliferation, metabolic conversion and genomic destabilization are important in the development of many chronic liver diseases, including alcoholic liver disease (ALD), hepatic fibrosis, NAFLD, HBV or HCV hepatitis and HCC [32-34]. What's more, some synergistic effects between virus infection, alcohol abuse, NAFLD and ER stress were found in the carcinogenesis of HCC [23]. In addition, ER stress can enhance cancer cell immune evasion and promote recurrence and metastasis by affecting the tumor microenvironment (TME) [35, 36]. ER stress-mediated UPR induces autophagy via IRE1a, PERK and ATF6 signaling channels and stimulates vascular endothelial growth factor(VEGF)secretion by macrophages, thereby promoting vasculogenesis in TME $[37,38]$. Studies to date have shown that ER stress plays a substantial role in regulating tumor cell fate through altered metabolic status and has emerged as a novel signaling target for the treatment of HCC. Inhibition of IRE1a, XBP1s and PERK expression could trigger tumor cell death under ER stressed conditions [24-26]. Proteasome inhibitor MLN2238 exacerbates ER stress and promotes cycle stagnation and apoptosis [39]. Sorafenib induces increased ER stress and activates cellular autophagy in HerpG2 cells [40]. Therefore, we hypothesized that aberrant expression of ER stress-related genes may have prognostic value for HCC patients.

In the current study, a novel seven-gene prognostic risk model based on ERSRGs was constructed and exhibited superior accuracy in forecasting the survival outcomes and 1-, 2-, 3-year survival rate of HCC patients in TCGA, ICGC and another cohort of individual clinical samples. More importantly, this feature was an independent risk factor for HCC patients when other clinical factors in the three cohorts were taken into account. In addition, significant effects of this feature on the immune microenvironment of $\mathrm{HCC}$ and the response to immune checkpoint inhibitors were investigated. Patients in high-risk score subgroup had significantly elevated ratios of Macrophages.M0 cells and increased TMB, PD1, PD-L1, CCL2, LAG3, CD276, CTLA4, CXCR4, IL1A, PD-L2, TGFB1, OX40 and CD137, indicating that ER stress could affect the immune microenvironment in $\mathrm{HCC}$ and immune checkpoint inhibitors (ICls) treatment was more effective for patients in high-risk subgroup. In addition, we explored the association between expression of genes in risk model and anticancer drug sensitivity in the CellMiner database, and identified 103 tumor-sensitive drugs that may be available for the treatment of HCC patients. Finally, to exploit the full potential of this risk model, a nomogram combining ER stress feature, TNM stage, age and gender was constructed and exhibited superior predictive performance. Therefore, our seven genetic risk models associated with ER stress can accurately predict survival outcome in HCC patients and facilitate the selection of the best individualized treatment. 
Of the seven genes in HCC patients, MAPT, NQ01, NGLY1, CDK5 and RNF186 expression were positively correlated with poor prognosis, while PON1 and PPARGC1A expression were negatively correlated with poor prognosis. Pathological aggregation of microtubule-associated protein tau (MAPT), a gene promotes microtubule protein assembly and microtubule stabilization, is the underlying mechanism that triggers endoplasmic reticulum ER stress [41]. Abnormal expression of MAPT could forecast the prognosis of patients in prostate cancer [42], glioma [43] and clear cell renal cell carcinoma [44]. NAD(P)H quinone oxidoreductase 1 (NQ01) plays an important role in cellular defense mechanisms against oxidative stress and ER stress and enhances the invasiveness and apoptosis evasion of hepatocellular carcinoma cells $[45,46]$. Aberrant expression of NGLY1 affects melanoma survival, tumor growth, and response to therapeutic agents in in vivo and in vitro and targeting NGLY1 is a novel anti-melanoma therapeutic modality [47]. Highly expressed Cyclin-Dependent Kinase 5 (CDK5) can promote the proliferation and oncogenicity of hepatocellular carcinoma cells by regulating the phosphorylation and stability of TPX2 [48], while inhibition of CDK5 enhances the sorafenib response in the treatment of HCC [49]. RING finger protein 186 (RNF186) inhibits the advancement of ER stress-mediated colorectal cancer by inhibiting NF-KB activity [50]. Serum PON1 level is a powerful prognostic factor and can be used to evaluate microvascular invasion in hepatocellular carcinoma [51]. PPARGC1A polymorphism favors the prognosis of patients by affecting the susceptibility to HCC [52]. However, relevant studies on MAPT, RNF186 and NQ01 in HCC are scarce and follow-up experiments are still needed to verify it.

In prior studies, a number of features have been identified for predicting survival outcomes in HCC patients [53-58]. Compared to these features, this seven-gene risk model has some new features: First, the signature is built based on ERSRG and ER stress is prevalent in HCC, which may provide new ideas for the treatment of HCC by targeting ER stress. Furthermore, the signature has been validated in a small clinical cohort by qRT-PCR analysis to guarantee its clinical relevance. Admittedly, our study has some limitations. The diversity and individual variability of HCC patients may reduce the performance of this feature. In addition, the small sample size limits the validation of the model, and future multicenter randomized controlled studies are needed to evaluate this signature. Finally, the expression and prognostic predictive role of these seven genes at the protein level and their specific mechanisms in HCC need to be further evaluated in the future by additional in vivo and in vitro experiments.

\section{Conclusions}

In summary, we constructed an ERSRG-based risk model in this study, which can effectively classify HCC patients for prognostic prediction and individualized immunotherapy targeting ER stress.

\section{Declarations}

\section{DATA AVAILABILITY STATEMENT}

We can find the datasets analyzed in this study in the https://xena.ucsc.edu and https://dcc.icgc.org/projects/LIRI-JP. 


\section{ETHICS STATEMENT}

This study was supported by the Ethics Committees of Zhengzhou University.

\section{AUTHOR CONTRIBUTIONS}

ZGH designed the study, downloaded, analyzed the data and wrote the manuscript and the manuscript.

The final manuscript has been approved by all authors.

\section{FUNDING}

No funding.

\section{CONSENT FOR PARTICIPATE}

Not applicable.

\section{CONSENT FOR PUBLICATION}

Consent for publication was obtained from all authors.

\section{DECLARATION OF COMPETING INTEREST}

The authors declare that there is no conflict of interests.

\section{ACKNOWLEDGEMENTS}

Not applicable.

\section{References}

1. Wang M, Kaufman RJ: Protein misfolding in the endoplasmic reticulum as a conduit to human disease. Nature 2016, 529(7586):326-335.

2. Schwarz DS, Blower MD: The endoplasmic reticulum: structure, function and response to cellular signaling. Cell Mol Life Sci 2016, 73(1):79-94.

3. Yukimoto A, Watanabe T, Sunago K, Nakamura Y, Tanaka T, Koizumi Y, Yoshida O, Tokumoto Y, Hirooka M, Abe M et al: The long noncoding RNA of RMRP is downregulated by PERK, which induces apoptosis in hepatocellular carcinoma cells. Sci Rep 2021, 11(1):7926.

4. Zhang Q, Guan G, Cheng P, Cheng W, Yang L, Wu A: Characterization of an endoplasmic reticulum stress-related signature to evaluate immune features and predict prognosis in glioma. $J \mathrm{Cell} \mathrm{Mol}$ Med 2021, 25(8):3870-3884.

5. Kapuy O, Márton M, Bánhegyi G, Vinod PK: Multiple system-level feedback loops control life-anddeath decisions in endoplasmic reticulum stress. FEBS Lett 2020, 594(6):1112-1123. 
6. Yu J, Li T, Liu Y, Wang X, Zhang J, Wang X, Shi G, Lou J, Wang L, Wang CC et al: Phosphorylation switches protein disulfide isomerase activity to maintain proteostasis and attenuate ER stress. Embo j 2020, 39(10):e103841.

7. Ron D, Walter P: Signal integration in the endoplasmic reticulum unfolded protein response. Nat Rev Mol Cell Biol 2007, 8(7):519-529.

8. Zhu H, Zhou H: Novel Insight into the Role of Endoplasmic Reticulum Stress in the Pathogenesis of Myocardial Ischemia-Reperfusion Injury. Oxid Med Cell Longev 2021, 2021:5529810.

9. Ajoolabady A, Wang S, Kroemer G, Klionsky DJ, Uversky VN, Sowers JR, Aslkhodapasandhokmabad $\mathrm{H}, \mathrm{Bi}$ Y, Ge J, Ren J: ER stress in Cardiometabolic Diseases: from Molecular Mechanisms to Therapeutics. Endocr Rev 2021.

10. Kratochvílová H, Mráz M, Kasperová BJ, Hlaváček D, Mahrík J, Laňková I, Cinkajzlová A, Matloch Z, Lacinová Z, Trnovská J et al: Different Expression of Mitochondrial and Endoplasmic Reticulum Stress Genes in Epicardial Adipose Tissue Depends on Coronary Atherosclerosis. Int J Mol Sci 2021, 22(9).

11. Liu Z, Zhu H, He C, He T, Pan S, Zhao N, Zhu L, Guan G, Liu P, Zhang Y et al: Nicorandil attenuates high glucose-induced insulin resistance by suppressing oxidative stress-mediated ER stress PERK signaling pathway. BMJ Open Diabetes Res Care 2021, 9(1).

12. Shrestha N, De Franco E, Arvan P, Cnop M: Pathological $\beta$-Cell Endoplasmic Reticulum Stress in Type 2 Diabetes: Current Evidence. Front Endocrinol (Lausanne) 2021, 12:650158.

13. Liu X, Chen A, Liang Q, Yang X, Dong Q, Fu M, Wang S, Li Y, Ye Y, Lan Z et al: Spermidine inhibits vascular calcification in chronic kidney disease through modulation of SIRT1 signaling pathway. Aging Cell 2021:e13377.

14. Wang YL, Lee YH, Hsu YH, Chiu IJ, Huang CC, Huang CC, Chia ZC, Lee CP, Lin YF, Chiu HW: The Kidney-Related Effects of Polystyrene Microplastics on Human Kidney Proximal Tubular Epithelial Cells HK-2 and Male C57BL/6 Mice. Environ Health Perspect 2021, 129(5):57003.

15. Minaei A, Sarookhani MR, Haghdoost-Yazdi H, Rajaei F: Hydrogen sulfide attenuates induction and prevents progress of the 6-hydroxydopamine-induced Parkinsonism in rat through activation of ATPsensitive potassium channels and suppression of ER stress. Toxicol App/ Pharmacol 2021:115558.

16. Gupta P, Tiwari S, Singh A, Pal A, Mishra A, Singh S: Rivastigmine attenuates the Alzheimer's disease related protein degradation and apoptotic neuronal death signalling. Biochem $J$ 2021, 478(7):14351451.

17. Jiang ZB, Xu C, Wang W, Zhang YZ, Huang JM, Xie YJ, Wang QQ, Fan XX, Yao XJ, Xie C et al: Plumbagin suppresses non-small cell lung cancer progression through downregulating ARF1 and by elevating CD8(+) cells. Pharmacol Res 2021:105656.

18. Jang H, Jun Y, Kim S, Kim E, Jung Y, Park BJ, Lee J, Kim J, Lee S, Kim J: FCN3 functions as a tumor suppressor of lung adenocarcinoma through induction of endoplasmic reticulum stress. Cell Death Dis 2021, 12(4):407. 
19. Chang CY, Pan PH, Wu CC, Liao SL, Chen WY, Kuan YH, Wang WY, Chen CJ: Endoplasmic Reticulum Stress Contributes to Gefitinib-Induced Apoptosis in Glioma. Int J Mol Sci 2021, 22(8).

20. Li C, Zhang K, Pan G, Ji H, Li C, Wang X, Hu X, Liu R, Deng L, Wang Y et al: Dehydrodiisoeugenol inhibits colorectal cancer growth by endoplasmic reticulum stress-induced autophagic pathways. $J$ Exp Clin Cancer Res 2021, 40(1):125.

21. Wei J, Yuan Y, Chen L, Xu Y, Zhang Y, Wang Y, Yang Y, Peek CB, Diebold L, Yang Y et al: ER-associated ubiquitin ligase HRD1 programs liver metabolism by targeting multiple metabolic enzymes. Nat Commun 2018, 9(1):3659.

22. Flessa CM, Kyrou I, Nasiri-Ansari N, Kaltsas G, Papavassiliou AG, Kassi E, Randeva HS: Endoplasmic Reticulum Stress and Autophagy in the Pathogenesis of Non-alcoholic Fatty Liver Disease (NAFLD): Current Evidence and Perspectives. Curr Obes Rep 2021.

23. Wei J, Fang D: Endoplasmic Reticulum Stress Signaling and the Pathogenesis of Hepatocarcinoma. Int J Mol Sci 2021, 22(4).

24. Pavlović N, Calitz C, Thanapirom K, Mazza G, Rombouts K, Gerwins P, Heindryckx F: Inhibiting IRE1aendonuclease activity decreases tumor burden in a mouse model for hepatocellular carcinoma. Elife 2020, 9.

25. Wu S, Du R, Gao C, Kang J, Wen J, Sun T: The role of XBP1s in the metastasis and prognosis of hepatocellular carcinoma. Biochem Biophys Res Commun 2018, 500(3):530-537.

26. Vandewynckel YP, Laukens D, Bogaerts E, Paridaens A, Van den Bussche A, Verhelst X, Van Steenkiste $C$, Descamps B, Vanhove $C$, Libbrecht $L$ et al: Modulation of the unfolded protein response impedes tumor cell adaptation to proteotoxic stress: a PERK for hepatocellular carcinoma therapy. Hepatol Int 2015, 9(1):93-104.

27. Su L, Zhang G, Kong X: Prognostic Significance of Pregnancy Zone Protein and Its Correlation with Immune Infiltrates in Hepatocellular Carcinoma. Cancer Manag Res 2020, 12:9883-9891.

28. Zhang G: Expression and Prognostic Significance of BANF1 in Triple-Negative Breast Cancer. Cancer Manag Res 2020, 12:145-150.

29. Yang C, Huang X, Liu Z, Qin W, Wang C: Metabolism-associated molecular classification of hepatocellular carcinoma. Mol Oncol 2020, 14(4):896-913.

30. Gentles AJ, Newman AM, Liu CL, Bratman SV, Feng W, Kim D, Nair VS, Xu Y, Khuong A, Hoang CD et al: The prognostic landscape of genes and infiltrating immune cells across human cancers. Nat Med 2015, 21(8):938-945.

31. Reinhold WC, Sunshine M, Liu H, Varma S, Kohn KW, Morris J, Doroshow J, Pommier Y: CellMiner: a web-based suite of genomic and pharmacologic tools to explore transcript and drug patterns in the NCl-60 cell line set. Cancer Res 2012, 72(14):3499-3511.

32. Baiceanu A, Mesdom P, Lagouge M, Foufelle F: Endoplasmic reticulum proteostasis in hepatic steatosis. Nat Rev Endocrinol 2016, 12(12):710-722.

33. Lebeaupin C, Vallée D, Hazari Y, Hetz C, Chevet E, Bailly-Maitre B: Endoplasmic reticulum stress signalling and the pathogenesis of non-alcoholic fatty liver disease. J Hepatol 2018, 69(4):927-947. 
34. Kim JY, Garcia-Carbonell R, Yamachika S, Zhao P, Dhar D, Loomba R, Kaufman RJ, Saltiel AR, Karin M: ER Stress Drives Lipogenesis and Steatohepatitis via Caspase-2 Activation of S1P. Cel/ 2018, 175(1):133-145.e115.

35. Urra H, Dufey E, Avril T, Chevet E, Hetz C: Endoplasmic Reticulum Stress and the Hallmarks of Cancer. Trends Cancer 2016, 2(5):252-262.

36. Mahadevan NR, Rodvold J, Sepulveda H, Rossi S, Drew AF, Zanetti M: Transmission of endoplasmic reticulum stress and pro-inflammation from tumor cells to myeloid cells. Proc Natl Acad Sci U S A 2011, 108(16):6561-6566.

37. Xia SW, Wang ZM, Sun SM, Su Y, Li ZH, Shao JJ, Tan SZ, Chen AP, Wang SJ, Zhang ZL et al: Endoplasmic reticulum stress and protein degradation in chronic liver disease. Pharmacol Res 2020, 161:105218.

38. Cullen SJ, Fatemie S, Ladiges W: Breast tumor cells primed by endoplasmic reticulum stress remodel macrophage phenotype. Am J Cancer Res 2013, 3(2):196-210.

39. Augello G, Modica M, Azzolina A, Puleio R, Cassata G, Emma MR, Di Sano C, Cusimano A, Montalto G, Cervello M: Preclinical evaluation of antitumor activity of the proteasome inhibitor MLN2238 (ixazomib) in hepatocellular carcinoma cells. Cell Death Dis 2018, 9(2):28.

40. Zhou B, Lu Q, Liu J, Fan L, Wang Y, Wei W, Wang H, Sun G: Melatonin Increases the Sensitivity of Hepatocellular Carcinoma to Sorafenib through the PERK-ATF4-Beclin1 Pathway. Int J Biol Sci 2019, 15(9):1905-1920.

41. Gao YL, Wang N, Sun FR, Cao XP, Zhang W, Yu JT: Tau in neurodegenerative disease. Ann Trans/ Med 2018, 6(10):175.

42. Schroeder C, Grell J, Hube-Magg C, Kluth M, Lang D, Simon R, Höflmayer D, Minner S, Burandt E, Clauditz TS et al: Aberrant expression of the microtubule-associated protein tau is an independent prognostic feature in prostate cancer. BMC Cancer 2019, 19(1):193.

43. Zaman S, Chobrutskiy BI, Sikaria D, Blanck G: MAPT (Tau) expression is a biomarker for an increased rate of survival for low-grade glioma. Oncol Rep 2019, 41(2):1359-1366.

44. Han X, Sekino Y, Babasaki T, Goto K, Inoue S, Hayashi T, Teishima J, Sakamoto N, Sentani K, Oue N et al: Microtubule-associated protein tau (MAPT) is a promising independent prognostic marker and tumor suppressive protein in clear cell renal cell carcinoma. Urol Oncol 2020, 38(6):605.e609605.e617.

45. Yang Y, Zheng J, Wang M, Zhang J, Tian T, Wang Z, Yuan S, Liu L, Zhu P, Gu F et al: NQ01 promotes an aggressive phenotype in hepatocellular carcinoma via amplifying ERK-NRF2 signaling. Cancer Sci 2021, 112(2):641-654.

46. Zhou HZ, Zeng HQ, Yuan D, Ren JH, Cheng ST, Yu HB, Ren F, Wang Q, Qin YP, Huang AL et al: NQ01 potentiates apoptosis evasion and upregulates XIAP via inhibiting proteasome-mediated degradation SIRT6 in hepatocellular carcinoma. Cell Commun Signal 2019, 17(1):168.

47. Zolekar A, Lin VJT, Mishra NM, Ho YY, Hayatshahi HS, Parab A, Sampat R, Liao X, Hoffmann P, Liu $\mathrm{J}$ et al: Stress and interferon signalling-mediated apoptosis contributes to pleiotropic anticancer 
responses induced by targeting NGLY1. Br J Cancer 2018, 119(12):1538-1551.

48. Wang F, Zhao W, Gao Y, Zhou J, Li H, Zhang G, Guo D, Xie C, Li J, Yin Z et al: CDK5-mediated phosphorylation and stabilization of TPX2 promotes hepatocellular tumorigenesis. J Exp Clin Cancer Res 2019, 38(1):286.

49. Ardelt MA, Fröhlich T, Martini E, Müller M, Kanitz V, Atzberger C, Cantonati P, Meßner M, Posselt L, Lehr T et al: Inhibition of Cyclin-Dependent Kinase 5: A Strategy to Improve Sorafenib Response in Hepatocellular Carcinoma Therapy. Hepatology 2019, 69(1):376-393.

50. Ji Y, Tu X, Hu X, Wang Z, Gao S, Zhang Q, Zhang W, Zhang H, Chen W: The role and mechanism of action of RNF186 in colorectal cancer through negative regulation of NF-KB. Cell Signal 2020, 75:109764.

51. Ding GY, Zhu XD, Ji Y, Shi GM, Shen YH, Zhou J, Fan J, Sun HC, Huang C: Serum PON1 as a biomarker for the estimation of microvascular invasion in hepatocellular carcinoma. Ann Trans/ Med 2020, 8(5):204.

52. Zhang S, Jiang J, Chen Z, Wang Y, Tang W, Chen Y, Liu L: Relationship of PPARG, PPARGC1A, and PPARGC1B polymorphisms with susceptibility to hepatocellular carcinoma in an eastern Chinese Han population. Onco Targets Ther 2018, 11:4651-4660.

53. Zhang BH, Yang J, Jiang L, Lyu T, Kong LX, Tan YF, Li B, Zhu YF, Xi AY, Xu X et al: Development and validation of a 14-gene signature for prognosis prediction in hepatocellular carcinoma. Genomics 2020, 112(4):2763-2771.

54. Long J, Chen P, Lin J, Bai Y, Yang X, Bian J, Lin Y, Wang D, Yang X, Zheng Y et al: DNA methylationdriven genes for constructing diagnostic, prognostic, and recurrence models for hepatocellular carcinoma. Theranostics 2019, 9(24):7251-7267.

55. Zhu G, Xia H, Tang Q, Bi F: An epithelial-mesenchymal transition-related 5-gene signature predicting the prognosis of hepatocellular carcinoma patients. Cancer Cell Int 2021, 21(1):166.

56. Cai JL, Zhu GQ, Du JX, Wang B, Wan JL, Xiao K, Dai Z: Identification and validation of a new gene signature predicting prognosis of hepatocellular carcinoma patients by network analysis of stemness indices. Expert Rev Gastroenterol Hepatol 2021:1-11.

57. Cai J, Tong Y, Huang L, Xia L, Guo H, Wu H, Kong X, Xia Q: Identification and validation of a potent multi-mRNA signature for the prediction of early relapse in hepatocellular carcinoma. Carcinogenesis 2019, 40(7):840-852.

58. Liu GM, Xie WX, Zhang CY, Xu JW: Identification of a four-gene metabolic signature predicting overall survival for hepatocellular carcinoma. J Cell Physiol 2020, 235(2):1624-1636.

\section{Figures}


A

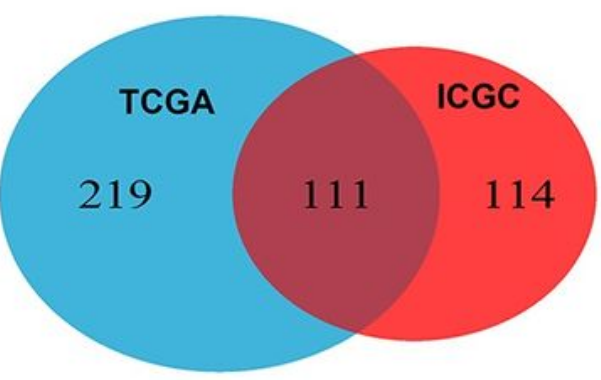

B
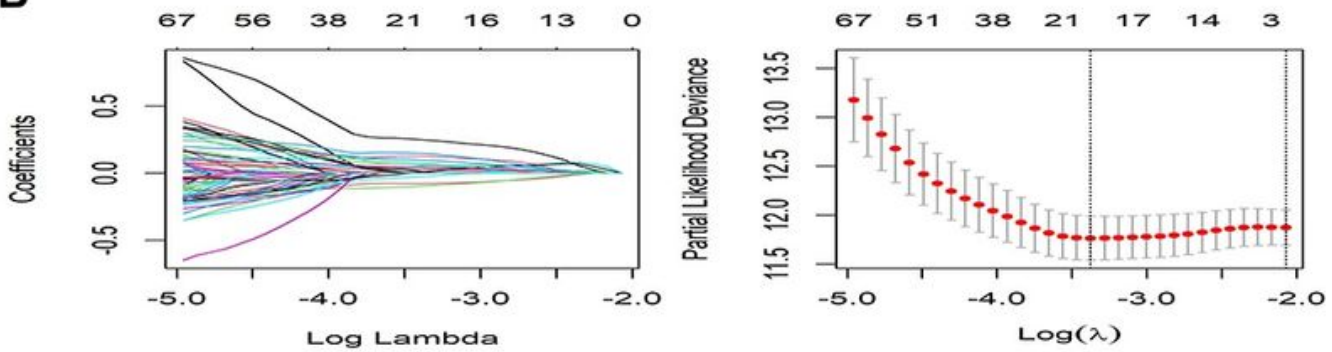

C

Log Lambda

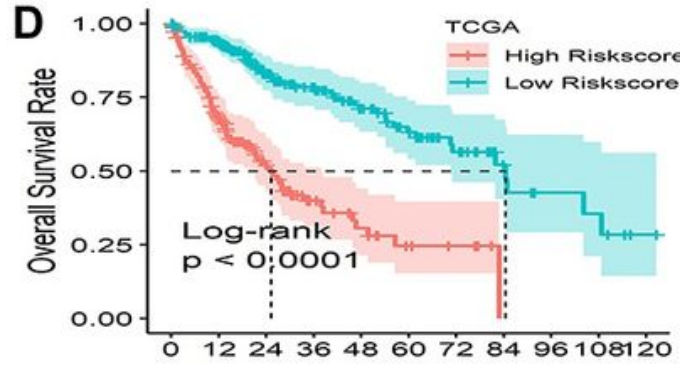

E
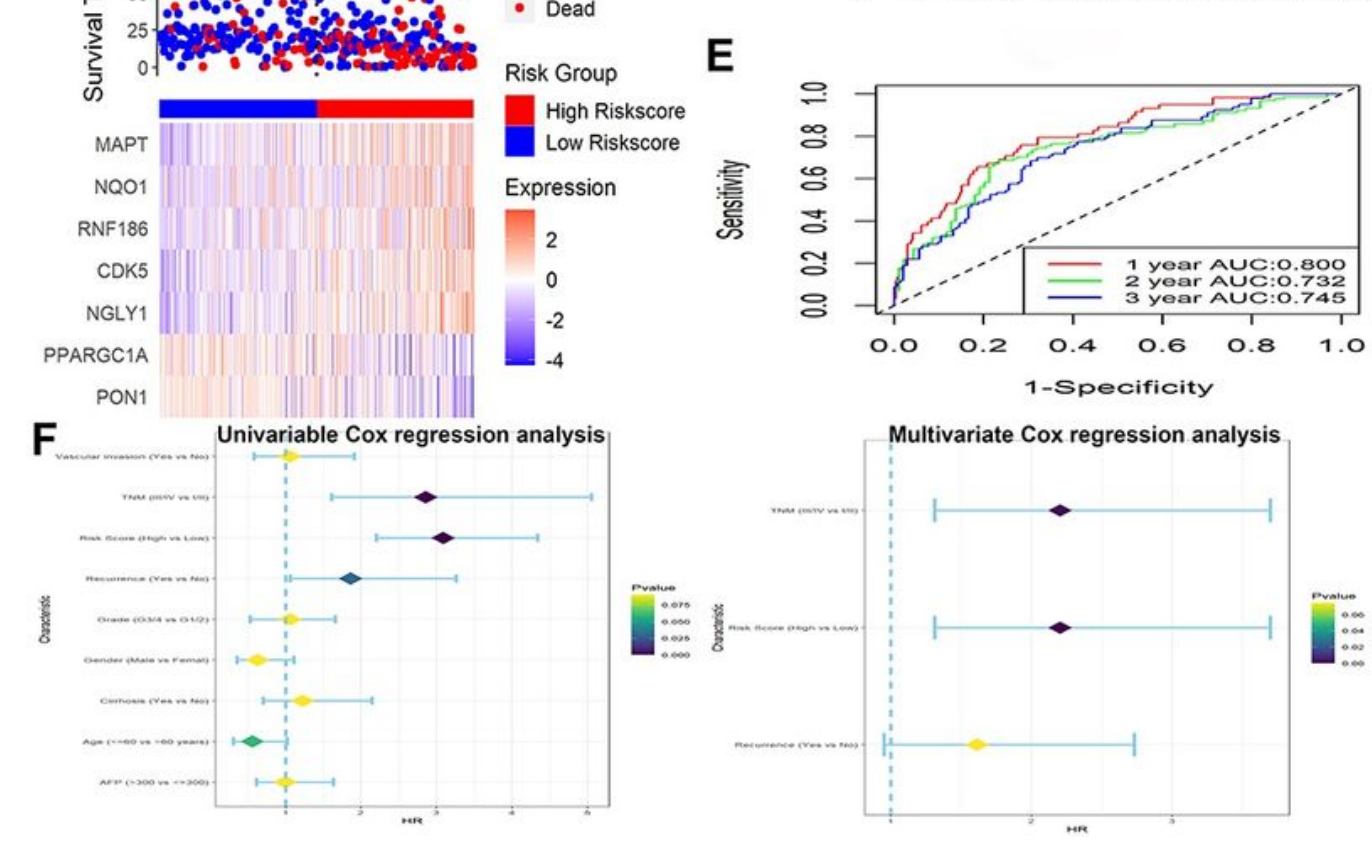

Risk Group
High Riskscore
Low Riskscore

MAPT Expression

RNF186

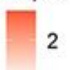

CDK5

$$
\text { NGLY1 }
$$

PPARGC1A

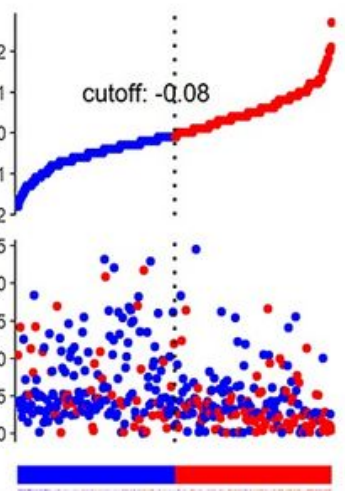

- High Riskscore

- Low Riskscore

- Alive

- Dead

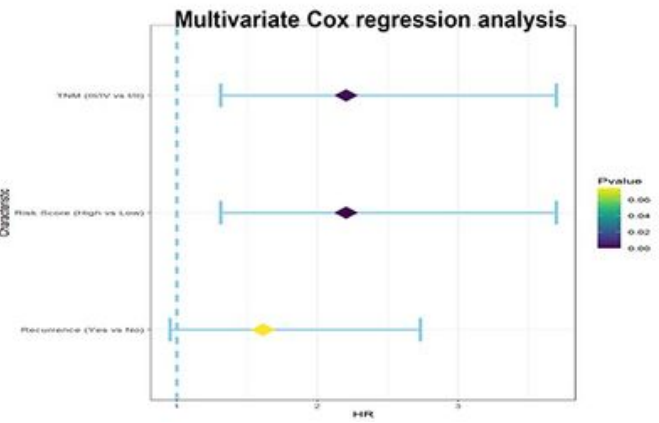

\section{Figure 1}

Development and survival examination of seven-gene signature in TCGA. (A) Identification of overlapping prognostic ERSRGs in TCGA and ICGC datasets. (B) The parameter selection in the LASSO-Cox analysis was adjusted by 10 cross-validations. (C) Distribution of risk scores, OS status, and gene expression profiles. (D) Kaplan-Meier survival plot. (E) Characteristics of predicted 1-, 2-, and 3-year OS rates in ROC analysis. (F) Forest plot showing the results of univariate (left) and multivariate (right) Cox analysis. 


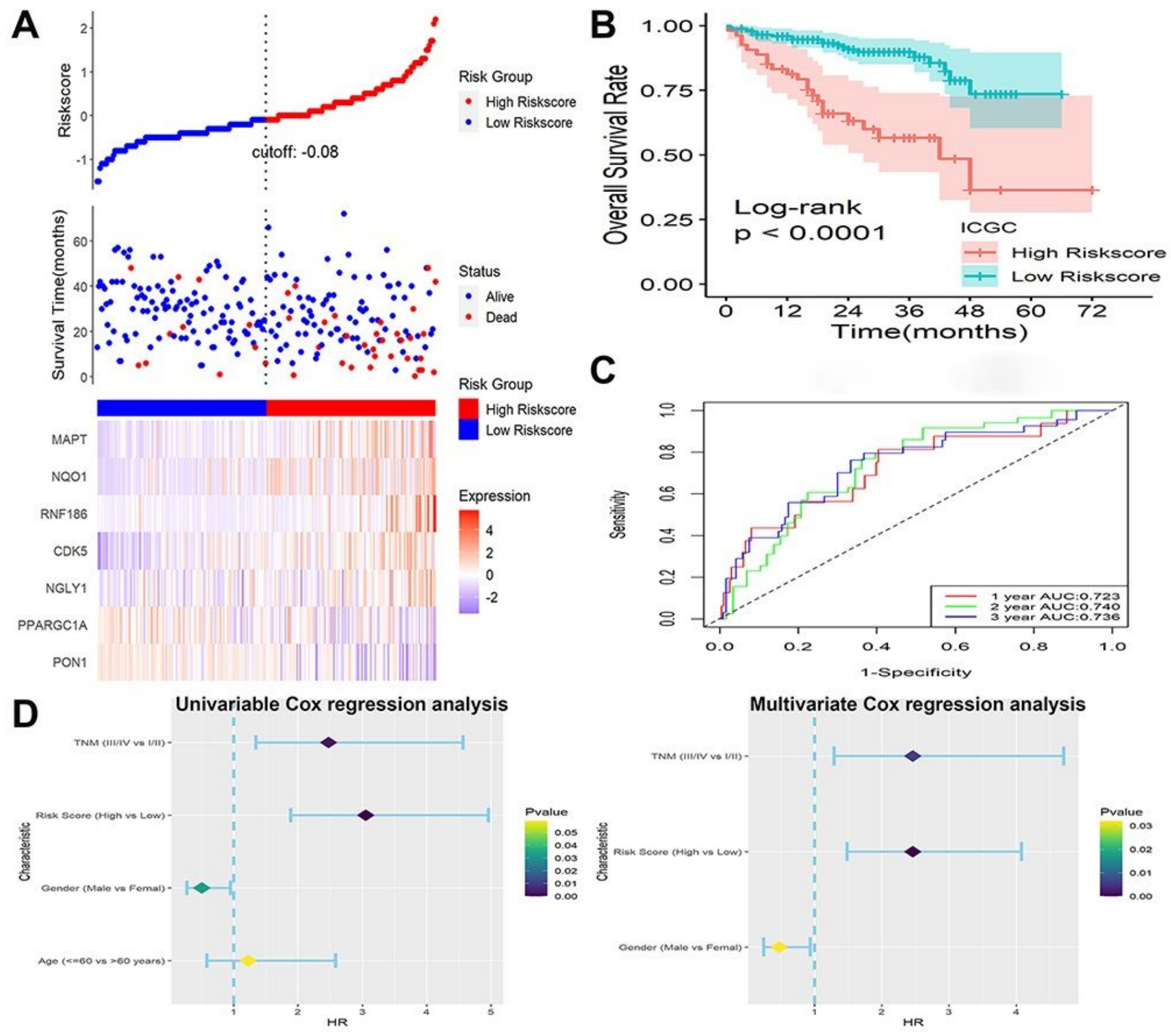

Figure 2

Validation of this feature in ICGC cohort. (A) Distribution of risk scores, OS status, and gene expression profiles. (B) Kaplan-Meier survival plot. (C) Characteristics of predicted 1-, 2-, and 3-year OS rates in ROC analysis. (D) Forest plot showing the results of univariate (left) and multivariate (right) Cox analysis. 

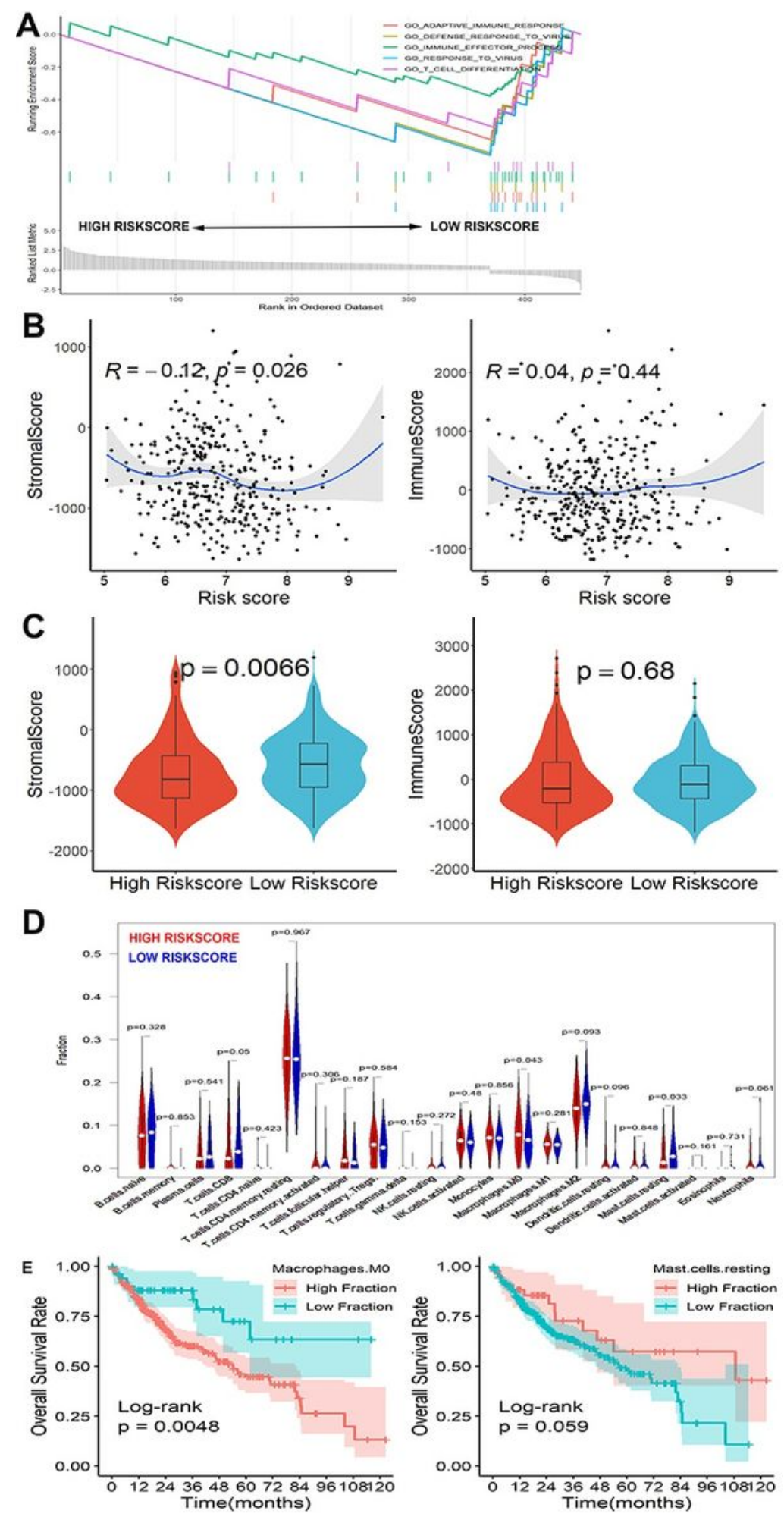

\section{Figure 3}

Functional analysis, immune status calculation and Immune infiltrates analysis. (A) GSEA based on the seven-gene signature. (B) Correlation analysis of risk scores, immune scores and stromal scores. (C) Difference analysis of immune scores and stromal scores in two subgroups. (D) Violin plot showing the abundance differentiation of 22 TIICs in two subgroups. (E) Kaplan-Meier survival plot for the two TIICs. 

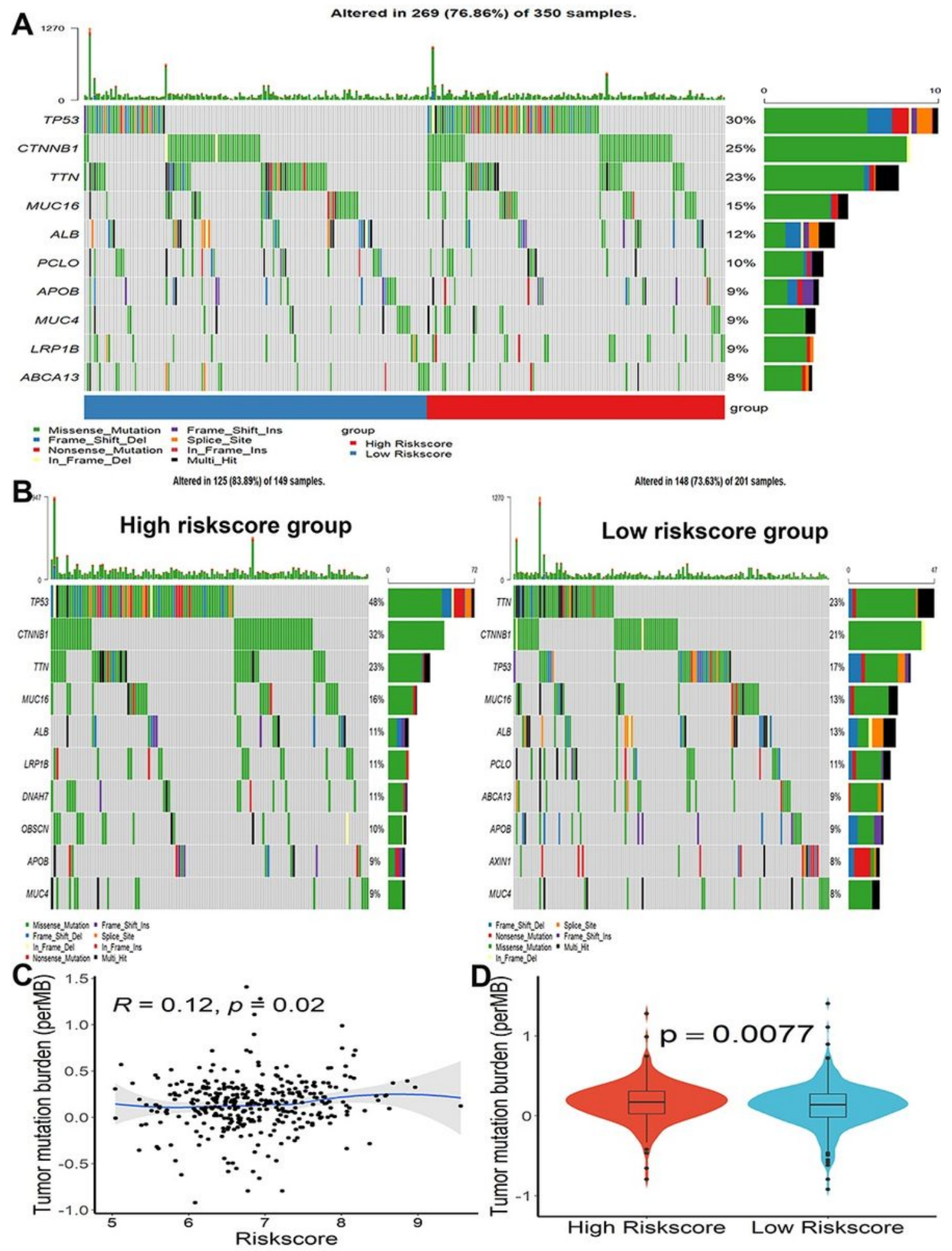

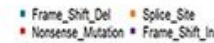

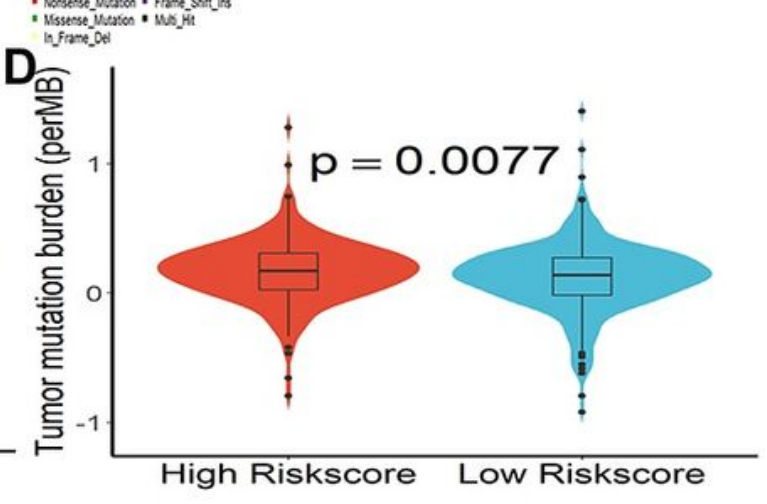

\section{Figure 4}

Somatic mutation and TMB analysis. (A) Mutation status of HCC patients in the TCGA cohort. (B) Tumor maps of mutated genes in two subgroups. (C) Correlation analysis of risk scores and TMB. (D) Differential analysis of TMB in the two subgroups. 

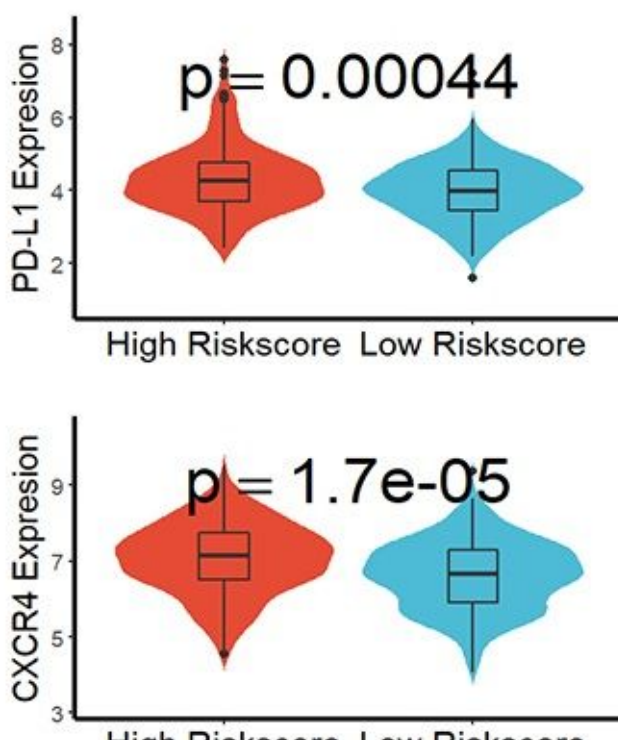

High Riskscore Low Riskscore
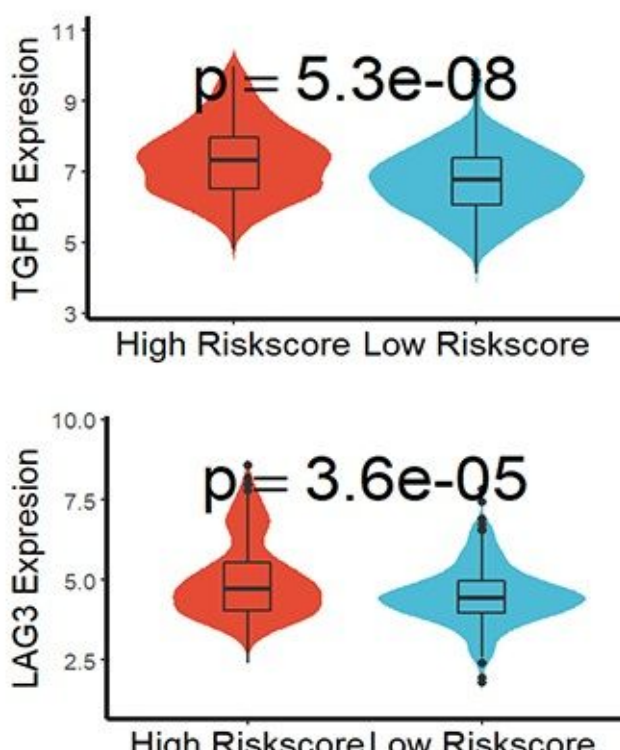

High Riskscore Low Riskscore
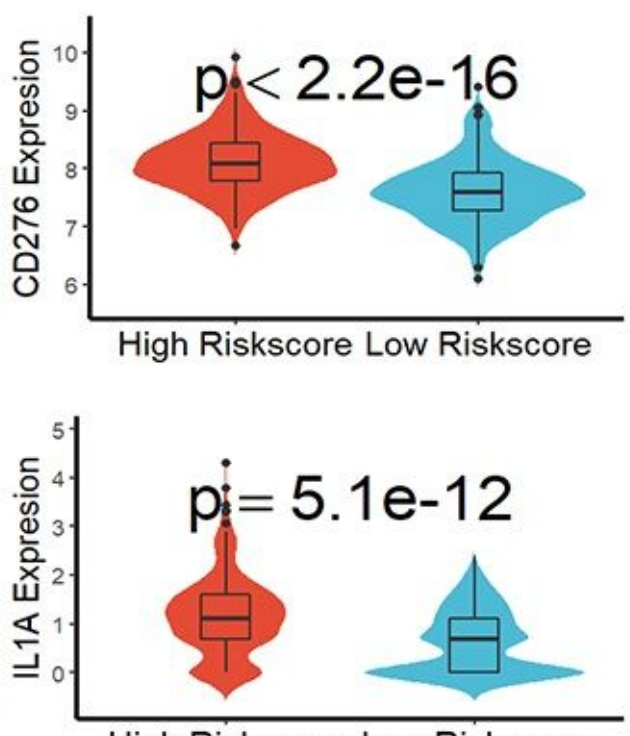

High Riskscore Low Riskscore

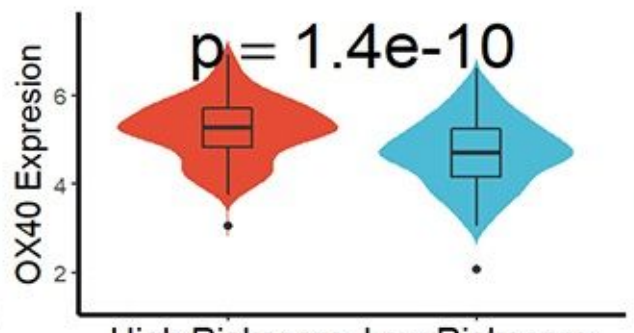

High Riskscore Low Riskscore

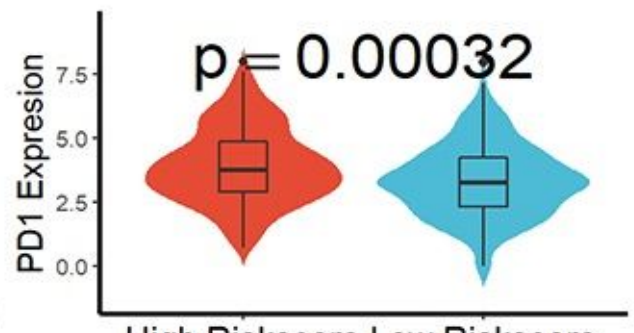

High Riskscore Low Riskscore

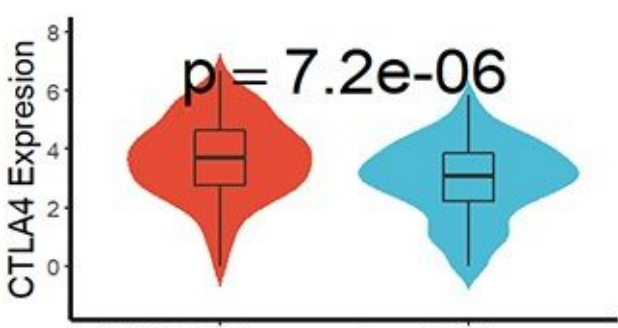

High Riskscore Low Riskscore

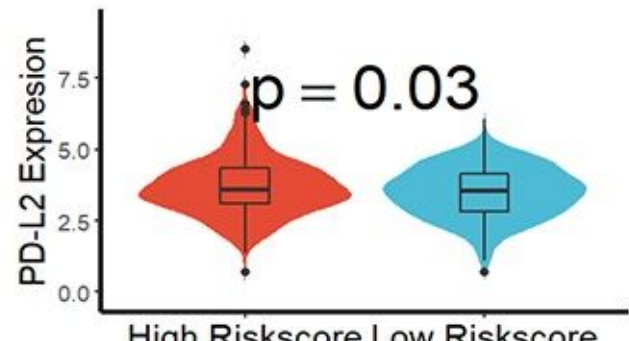

High Riskscore Low Riskscore
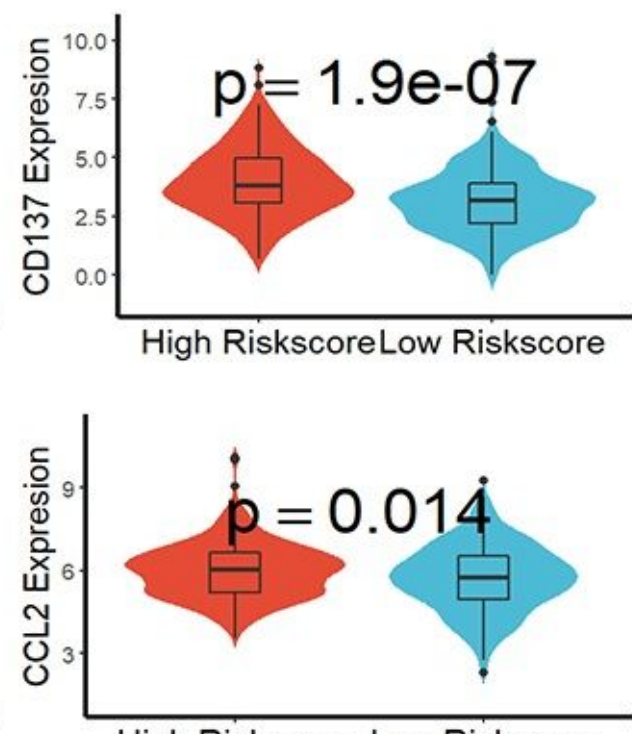

High Riskscore Low Riskscore

Figure 5

Levels of immune checkpoint genes in two subgroups. 


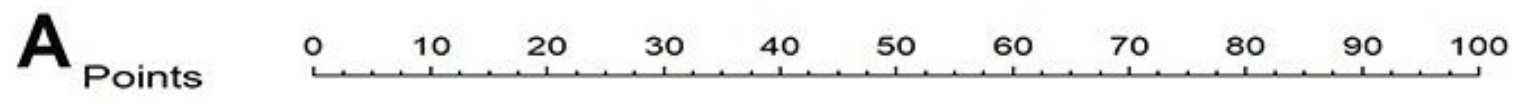

Age

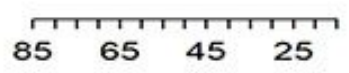

Gender

$$
\text { male }
$$

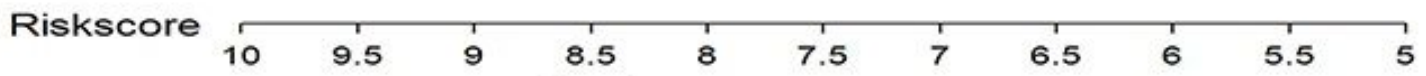

TNM.stage $\underset{\text { Stage IV Stage III Stage II }}{\text { Stage I }}$

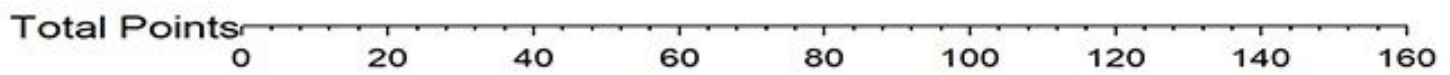

\section{Linear Predictor}

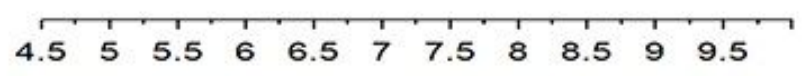

1-year Survival Probability

$$
0.40 .50 .60 .70 .8 \quad 0.9
$$

2-year Survival Probability

$0.40 .50 .60 .7 \quad 0.8 \quad 0.9$

3-year Survival Probability

$$
0.40 .50 .60 .70 .8 \quad 0.9
$$

B
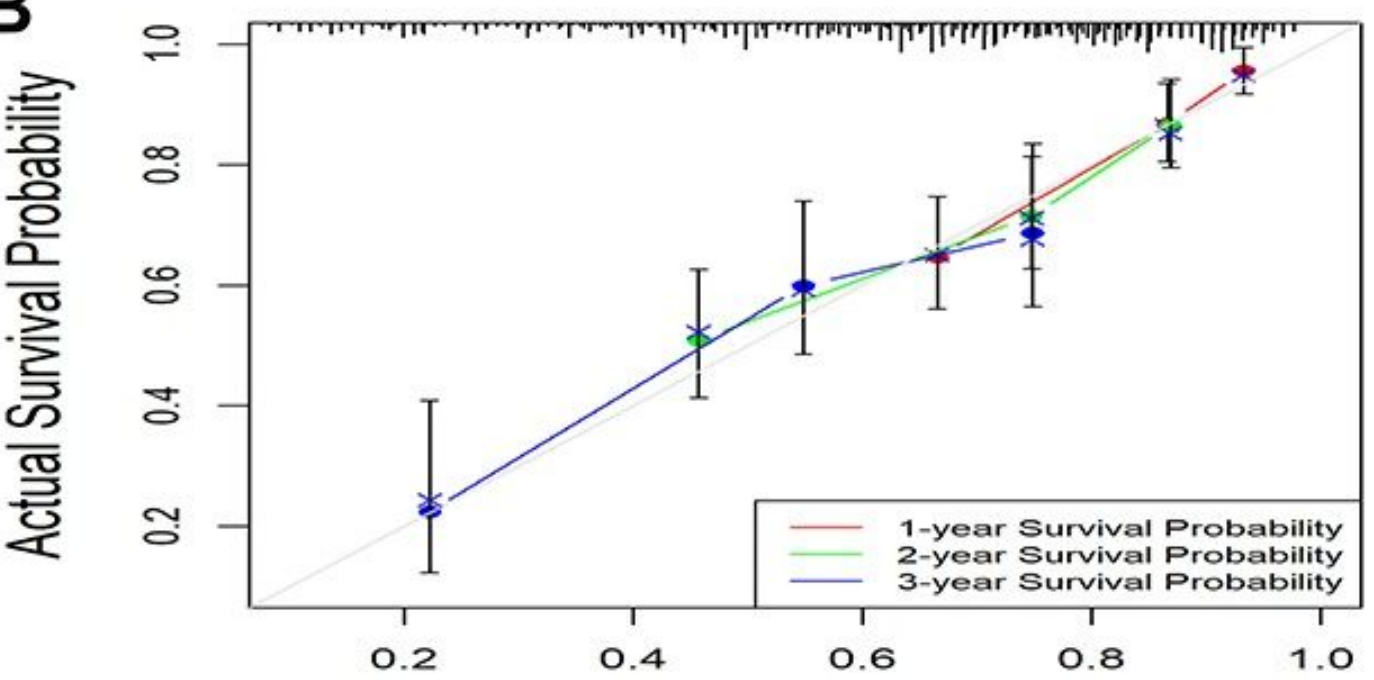

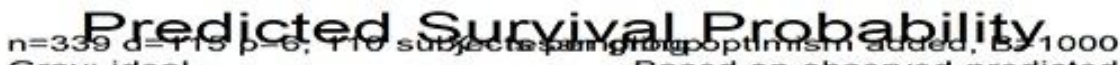
Gray: ideal

Based on observed-predicted

\section{Figure 6}

The predictive significance of the signatures was verified in the nomogram model. (A) Nomogram combining the seven genetic signatures. (B) Calibration plots of 1-, 2-, and 3-year survival probabilities. 
PON1, Nelarabine

Cor $=0.792, p<0.001$

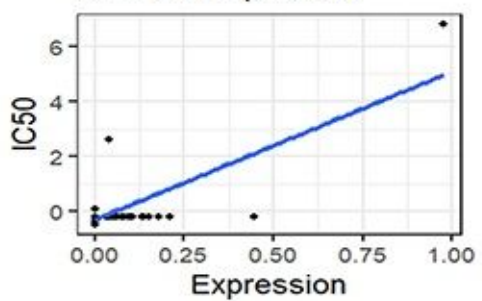

PON1, Dexamethason $\epsilon$ Cor $=0.551, p<0.001$

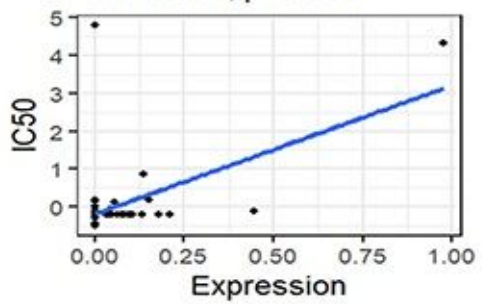

PON1, PX-316

Cor $=0.522, p<0.001$

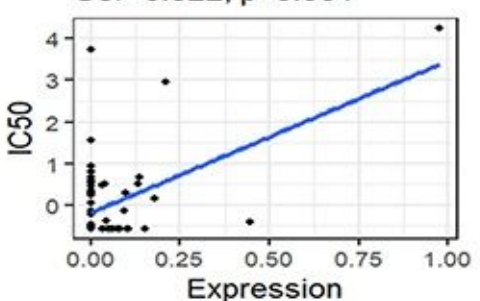

NQO1, 7-Hydroxystal Cor $=-0.495, p<0.001$

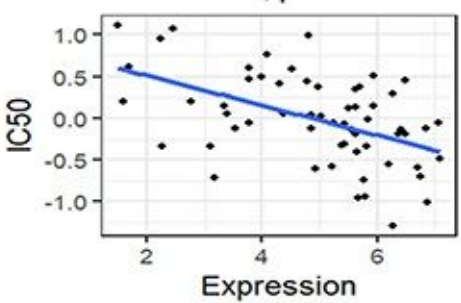

NQO1, Imexon

Cor $=-0.629, p<0.001$

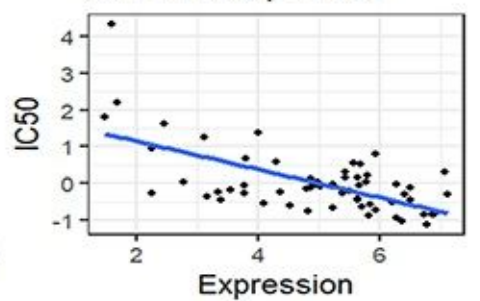

PON1, Chelerythrine Cor $=0.549, p<0.001$

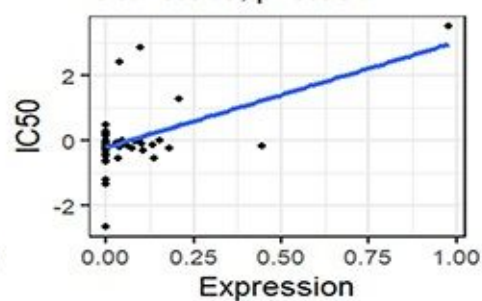

NQO1, DMAPT

Cor $=-0.516, p<0.001$

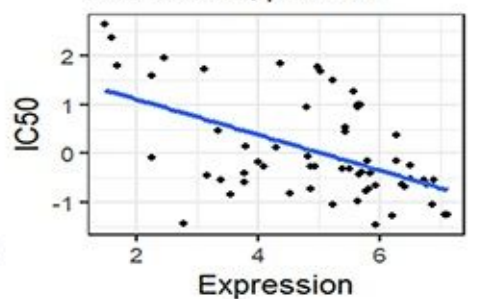

NQO1, EC-330

Cor $=-0.494, p<0.001$

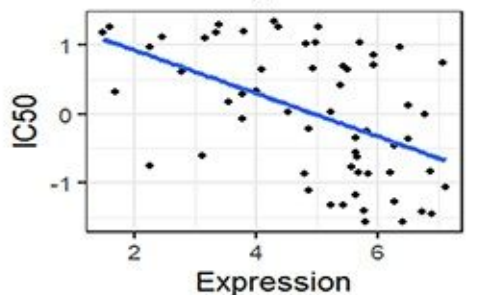

PON1, Fluphenazine Cor $=0.609, p<0.001$

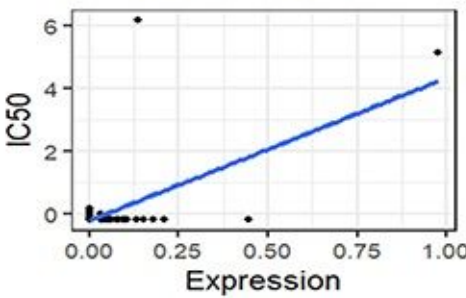

NGLY1, Chelerythrine Cor $=0.531, p<0.001$

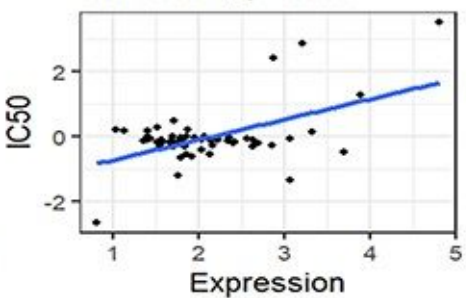

NQO1, DACARBAZINI Cor $=-0.504, p<0.001$

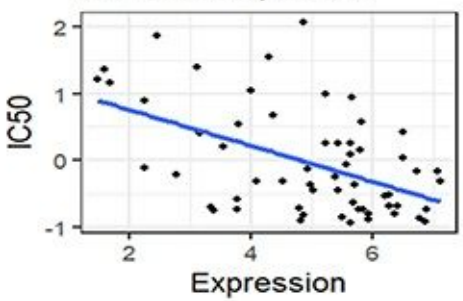

PON1, ZM-336372

Cor $=0.482, p<0.001$

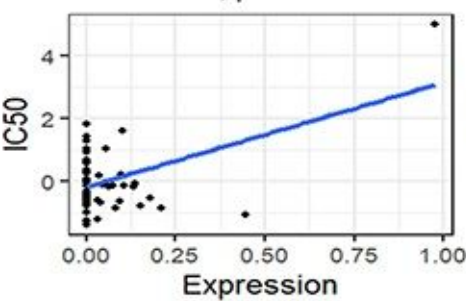

NQO1, Arsenic trioxide Cor $=-0.596, p<0.001$

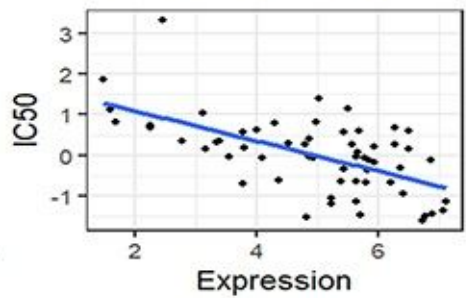

NGLY1, Nelarabine Cor $=0.524, p<0.001$

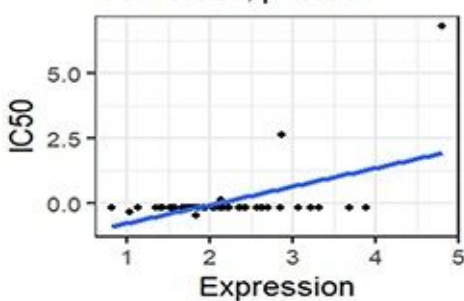

NQO1, Carmustine Cor $=-0.502, p<0.001$

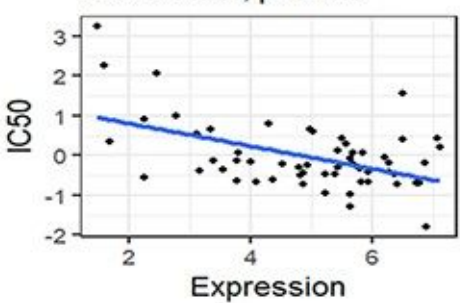

NGLY1, INK-128

Cor $=-0.481, p<0.001$

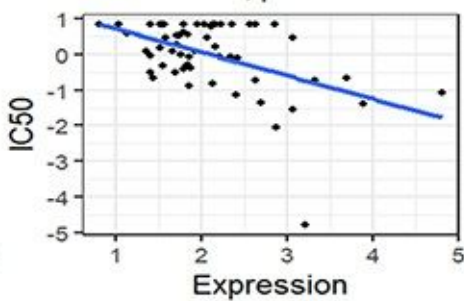

Figure 7

Correlation analysis of mRNA molecules in our risk model and the top 16 most significant tumor-sensitive drugs.
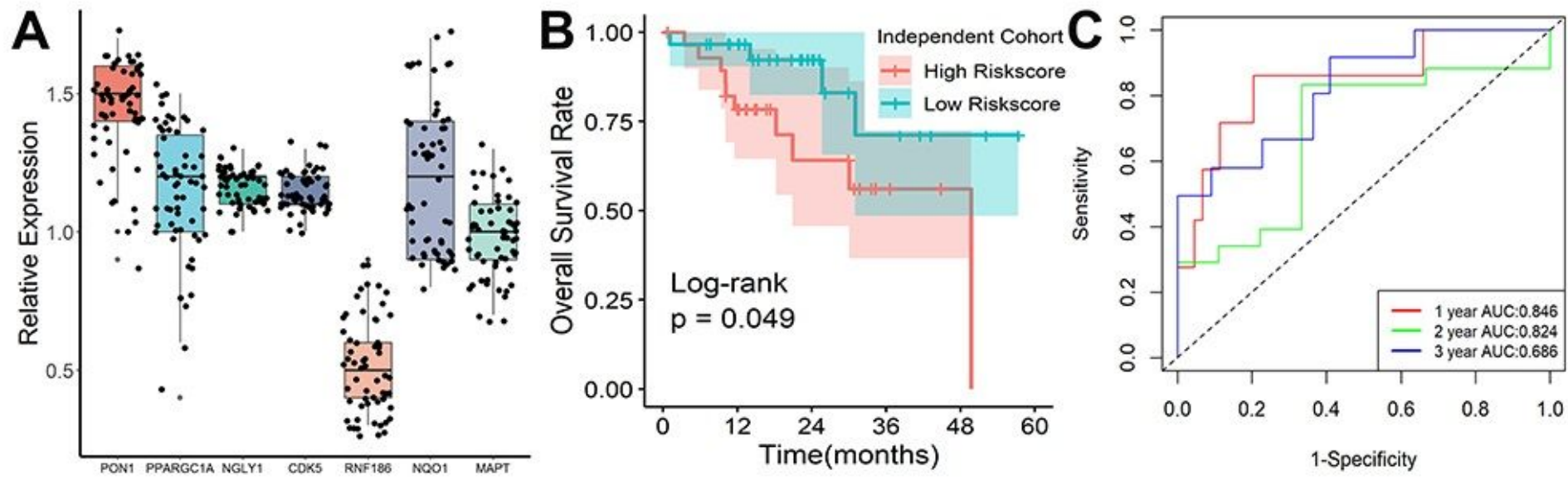
Figure 8

Validation of this feature in an independent clinical cohort. (A) Expression levels of mRNA molecules in our risk model. (B) Kaplan-Meier survival plot. (C) Characteristics of predicted 1-, 2-, and 3-year OS rates in ROC analysis.

\section{Supplementary Files}

This is a list of supplementary files associated with this preprint. Click to download.

- ERrelatedgenes.csv

- FigureS1.tif

- FigureS2.tif

- FigureS3.tif

- TableS12.docx

- TableS3.xIsx 